\title{
Protective effects of tetrahydropalmatine against ketamine-induced learning and memory injury via antioxidative, anti-inflammatory and anti-apoptotic mechanisms in mice
}

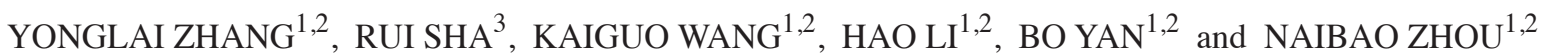 \\ ${ }^{1}$ Department of Anesthesiology, Shandong Cancer Hospital Affiliated to Shandong University, \\ Shandong Academy of Medical Sciences, Jinan, Shandong 250117; ${ }^{2}$ Department of Anesthesiology, \\ Shandong Academy of Medical Sciences, Jinan, Shandong 250200; ${ }^{3}$ Department of Anesthesiology, \\ Oncology Ward, Chinese Medicine Hospital in Shandong Province, Jinan, Shandong 250117, P.R. China
}

Received August 22, 2016; Accepted August 31, 2017

DOI: $10.3892 / \mathrm{mmr} .2018 .8700$

\begin{abstract}
Tetrahydropalmatine exerts numerous pharmacological activities, including analgesic and narcotic effects; anti-arrhythmic, blood pressure lowering and cardioprotective effects; protective effects against cerebral ischemia-reperfusion injury; inhibition of platelet aggregation; prevention of ulcerative diseases and inhibition of gastric acid secretion; antitumor effects; and beneficial effects on the withdrawal symptoms associated with drug addiction. The present study aimed to investigate the protective effects of tetrahydropalmatine against ketamine-induced learning and memory impairment in mice. The Morris water maze test and open field test were used to analyzed learning and memory impairment in mice. ELISA kits and western blotting were used to analyze oxidative stress, inflammation factors, caspease-3 and caspase-9, iNOS, glial fibrillary acidic protein (GFAP), glial cell-derived neurotrophic factor (GDNF), cytochrome $\mathrm{c}$ and phospholipase C (PLC)- $\gamma 1$ protein expression. The results demonstrated that tetrahydropalmatine treatment significantly decreased escape latency in the learning phase and increased the number of platform site crossings in ketamine-induced mice. In addition, tetrahydropalmatine significantly inhibited oxidative stress, inflammation and acetylcholinesterase activity, and decreased acetylcholine levels in ketamine-induced mice. Tetrahydropalmatine also suppressed iNOS protein expression, weakened caspase-3 and caspase- 9 activation, inhibited nuclear factor $-\kappa \mathrm{B}$, glial fibrillary acidic
\end{abstract}

Correspondence to: Dr Yonglai Zhang, Department of Anesthesiology, Shandong Cancer Hospital Affiliated to Shandong University, Shandong Academy of Medical Sciences, 440 Jiyan Road, Jinan, Shandong 250117, P.R. China

E-mail: yijiangzhimei@126.com

Key words: tetrahydropalmatine, ketamine, learning and memory impairment, glial fibrillary acidic protein, glial cell-derived neurotrophic factor, cytochrome $c$, phospholipase C- $\gamma 1$ protein, cytochrome $c$ and phospholipase $C-\gamma 1$ protein expression, and induced glial cell-derived neurotrophic factor protein expression in ketamine-induced mice. Taken together, these results indicated that tetrahydropalmatine may protect against ketamine-induced learning and memory impairment in mice via antioxidative, anti-inflammatory and anti-apoptotic mechanisms. The present study provided an experimental basis for the clinical application of tetrahydropalmatine to reduce the severe side effects associated with ketamine therapy in future studies.

\section{Introduction}

With the rapid development of medical technology, the complexity of pediatric surgery has increased, as has the use of general anesthesia (1). It is well known in pediatric surgery that inhaled or intravenously administered general anesthesia results in the inhibition of synaptic transmission in the brain, thus resulting in a temporary loss of consciousness, which is reversible. Subsequently, the anesthetic is excreted from the body by rapid metabolism or in its original form, without resulting in long-term damage to the developing brain; therefore, if there are no factors leading to cerebral hypoxia during anesthesia, the intellectual development of infants and young children administered anesthesia will not be affected $(1,2)$. However, recent research has indicated that the effects of anesthesia on central nervous system development is not as simple as originally presumed, and the administration of general anesthesia during the peak of nervous system development can induce long-term neurobehavioral alterations and cognitive function defects (3).

Ketamine (chemical formula, $\mathrm{C}_{13} \mathrm{H}_{16} \mathrm{ClNO}$ ), which is a white crystalline powder at room temperature, is a similar compound to phenylcyclidine and is a non-competitive antagonist of the N-methyl-D-aspartate (NMDA) receptor (3). Ketamine can selectively act on the neural pathway, and can block the pain pathway, thus resulting in improved analgesia; therefore, ketamine is often used as an anesthetic in minor surgery, pediatric examination or diagnostic procedures. In addition, ketamine inhibits the functional activities of the new 
cortical in relation to the hypothalamus, stimulates the reward pathways of the limbic system, and has the potential to induce psychological dependence (3). Ketamine initiates the generation of strong euphoria, and continuous use for recreational purposes can seriously damage the cognitive function of the nervous system and mental health (4).

At present, although it has been confirmed by numerous studies that chronic exposure to ketamine leads to persistent cognitive impairment, such as in learning and memory, there are relatively few studies regarding potential therapeutic intervention, as the precise biological mechanism underlying chronic ketamine-induced cognitive impairment remains unclear $(5,6)$.

Traditional Chinese medicines, including Corydalis Tuber, are believed to possess numerous functions, including activating blood circulation, dissipating stasis and regulating Qi. In addition, traditional Chinese medicines may prevent platelet aggregation, dilate small blood vessels and improve microcirculation; these compounds are widely used to clinically treat ischemic cerebrovascular disease (7). Tetrahydropalmatine (Fig. 1) is widely present in numerous plants and plant extracts, such as the following: Yuan Hu, Stephania, Hierophis viridiflavus, yellow vine and Decumbent Corydalis Tuber. Tetrahydropalmatine is also known as Rotundine (8). Tetrahydropalmatine is an alkaloid that can be extracted from the Corydalis genus from the Papaveraceae plant family. According to previous studies, tetrahydropalmatine possesses numerous pharmacological activities, including analgesic, anti-cerebral ischemia, anti-arrhythmic and anti-ischemic effects; therefore, tetrahydropalmatine is used as a sedative, analgesic, and tranquilizer in clinical practice $(8,9)$. The present study aimed to investigate whether tetrahydropalmatine protects against ketamine-induced learning and memory impairment in mice.

\section{Materials and methods}

Animals and drug administration. Male C57BL/6 mice (age, 6-8 weeks; weight, 20 \pm 2 g) were purchased from Experimental Animal Center of Shandong University, and housed under the following conditions: $23 \pm 2{ }^{\circ} \mathrm{C}, 50 \pm 5 \%$ humidity, under a 12-h light/dark cycle, and were given ad libitum access to standard chow and water. A total of 38 mice were divided into five groups ( $n=6-8 /$ group): Control group ( $n=6 /$ group), model group (n=8/group), and 20, 40 and $80 \mathrm{mg} / \mathrm{kg}$ tetrahydropalmatine groups ( $\mathrm{n}=8 /$ group). In model or 20,40 and $80 \mathrm{mg} / \mathrm{kg}$ tetrahydropalmatine groups, mice were intraperitoneally (i.p.) injected with $80 \mathrm{mg} / \mathrm{kg}$ of ketamine. Then, 20,40 and $80 \mathrm{mg} / \mathrm{kg}$ tetrahydropalmatine groups were i.p. injected at $10 \mathrm{ml} / \mathrm{kg}$ body weight for 1 week. In control group, mice were i.p. injected with normal saline. The present study was approved by the Animal Administration Committee of Shandong Tumor Combat Research Institute (Jinan, China) and were performed according to the Guidelines for the Care and Use of Laboratory Animals published by the National Institutes of Health (10).

Morris water maze test and open field test. After 1 week of treatment with tetrahydropalmatine, mice underwent a spatial learning test. A Morris water maze (120-cm diameter; Shenzhen Rui Wode Life Technology Co., Ltd., Shenzhen, China) was placed at $60 \mathrm{~cm}$ depth; water temperature was maintained at $21-23^{\circ} \mathrm{C}$. A cylindrical platform (14-cm diameter) was placed into the maze $(1-1.5 \mathrm{~cm}$ below the water surface) $35 \mathrm{~cm}$ from the pool wall. Mice were allowed to remain for $90 \mathrm{sec}$ and the time to find the target recorded. The water maze test was observed for 5 days. At the end of training, the pool was cleaned to eliminate olfactory cues. For the open field test, an open field $(36 \times 36 \mathrm{~cm})$ was created as follows: An area of tiled floor with high plywood planks $(40 \mathrm{~cm})$ was divided into 25 equal squares during the open field test. Mice were placed in the center of the open field and the path length of every mice was recorded for $1 \mathrm{~min}$.

Biochemical analysis of brain tissue. Following treatment with tetrahydropalmatine, mice were sacrificed using $35 \mathrm{mg} / \mathrm{kg}$ pentobarbital sodium and the hippocampus was dissected from each mouse onto an ice-cold plate. Proteins were extracted from the hippocampal samples using an RIPA assay (Beyotime Institute of Biotechnology, Haimen, China) and were used to measure glutathione (GSH)-peroxidase (GSH-PX; A005), GSH (A006-2), superoxide dismutase (SOD; A001-1-1), malondialdehyde (MDA; A003-1), tumor necrosis factor (TNF)- $\alpha$ (H052), interleukin (IL)-1 $\beta$ (H002), IL-6 (H007) and acetylcholine (ACh; A105-1) levels, as well as acetylcholinesterase (AChE; A024), caspase-3 (G015) and caspase-9 activity (G018) using commercially available ELISA kits (Nanjing Jiancheng Bioengineering Research Institute, Nanjing, China) according to the manufacturer's protocols. The absorbance was then measured using an Infinite M200 PRO plate reader (Tecan Group Ltd., Männedorf, Switzerland) at 450 or $405 \mathrm{~nm}$.

Western blotting. The hippocampus was dissected from each mouse onto an ice-cold plate. Proteins were extracted from the hippocampal samples using an extraction kit (Beyotime Institute of Biotechnology) and protein concentration was determined using BCA assay (Beyotime Institute of Biotechnology) and an ultraviolet spectrophotometer (UV-1601; Shimadzu Corporation, Kyoto, Japan). Subsequently, $50 \mu \mathrm{g}$ total protein was size-fractionated by $10 \%$ SDS-PAGE and was immunoblotted onto polyvinylidene fluoride membranes. The membranes were blocked with 5\% non-fat milk in TBST for $1 \mathrm{~h}$ at $37^{\circ} \mathrm{C}$ and then incubated with antibodies against iNOS (13120; 1:2,000), GFAP (12389; 1:2,000), GDNF (3897, 1:2,000), cytochrome c (11940; 1:2,000), PLC- $\gamma 1$ (5690; 1:2,000) and GAPDH (5174; 1:1,000; all from Cell Signaling Technology, Inc., Danvers, MA, USA) at $4^{\circ} \mathrm{C}$ overnight. After washing with Tris-buffered saline containing $0.01 \%$ Tween-20, the membranes were incubated with biotinylated goat anti-rabbit IgG-HRP secondary antibody (sc-2030; 1:5,000; Santa Cruz Biotechnology, Inc., Dallas, TX, USA) for $1 \mathrm{~h}$ at $37^{\circ} \mathrm{C}$, and were visualized using an enhanced chemiluminescence kit (Amersham; GE Healthcare Life Sciences, Little Chalfont, UK) and analyzed using Image-ProPlus software version 6.0 (Media Cybernetics, Inc., Rockville, MD, USA).

Statistical analysis. Data are presented as the mean \pm standard error of the mean $(n=3)$ using SPSS version 17.0 (SPSS, Inc., Chicago, IL, USA) and were analyzed using two-way analysis of variance and Tukey's post hoc test with repeated measures. $\mathrm{P}<0.05$ was considered to indicate a statistically significant difference. 


\section{Results}

Protective effects of tetrahydropalmatine against ketamine-induced learning and memory impairment in mice. Mice treated with ketamine exhibited increased escape latency and mean path length compared with the control mice (Fig. 2A and B). In addition, ketamine inhibited the percentage of time spent in the target quadrant and the number of times crossing the platform compared with the control mice (Fig. 2C and D). Conversely, tetrahydropalmatine $(80 \mathrm{mg} / \mathrm{kg})$ effectively inhibited the ketamine-induced increase in escape latency and mean path length, and reversed the ketamine-induced decrease in the percentage of time spent in the target quadrant and the number of times crossing the platform (Fig. 2A-D).

Protective effects of tetrahydropalmatine against ketamine-induced oxidative stress. To determine the effects of tetrahydropalmatine on ketamine-induced oxidative stress in mice, GSH-PX, GSH, SOD and MDA activities were measured using ELISA kits. The results demonstrated that there was a significant decrease in GSH-PX, GSH and SOD activities, and an increase in MDA content, in ketamine-induced mice compared with in the control mice (Fig. 3). However, treatment with tetrahydropalmatine significantly increased GSH-PX, GSH and SOD activities, and inhibited MDA activity, in ketamine-induced mice (Fig. 3).

Protective effects of tetrahydropalmatine against ketamine-induced inflammation. TNF- $\alpha$, IL-1 $\beta$ and IL-6 activities were examined using ELISA kits. As shown in Fig. 4, there was a significant increase in TNF- $\alpha$, IL-1 $\beta$ and IL-6 activities in ketamine-induced mice compared with in control mice. Tetrahydropalmatine significantly decreased TNF- $\alpha$, IL-1 $\beta$ and IL- 6 activities in ketamine-induced mice (Fig. 4).

Protective effects of tetrahydropalmatine against ACh levels and AChE activities in ketamine-induced mice. ACh levels and AChE activities were examined using ELISA kits. As presented in Fig. 5, AChE activity was induced and ACh levels were inhibited in ketamine-induced mice compared with control mice. Administration of tetrahydropalmatine significantly reduced ketamine-induced AChE activity and increased ACh levels in ketamine-induced mice (Fig. 5).

Effects of tetrahydropalmatine on iNOS, GDNF and NF-кB protein in ketamine-induced mice. The results of a western blot analysis revealed that ketamine significantly induced iNOS and $\mathrm{NF}-\kappa \mathrm{B}$ protein expression in mice compared with in the control group (Fig. 6A-C). Conversely, GDNF protein expression was significantly suppressed in the ketamine model group compared with in the control group (Fig. 6A and D). Compared with in the ketamine model group, treatment with tetrahydropalmatine significantly suppressed iNOS and $\mathrm{NF}-\kappa \mathrm{B}$ protein expression, and induced GDNF protein expression (Fig. 6).

Protective effects of tetrahydropalmatine against caspase-3 and caspase-9 activation in ketamine-induced mice.

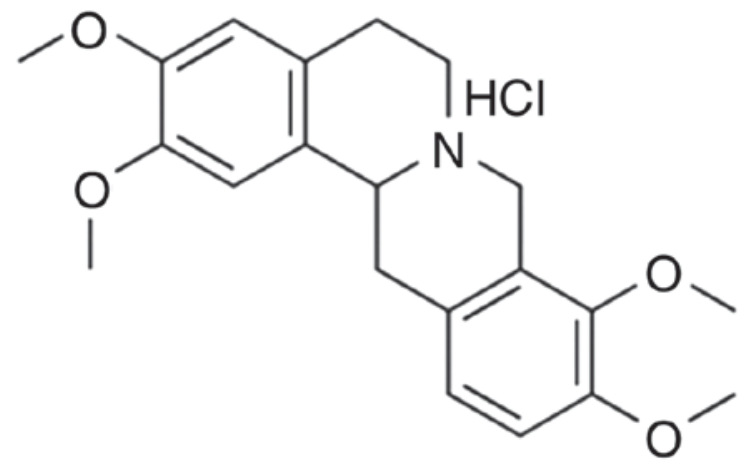

Figure 1. Constitutional formula of tetrahydropalmatine.

Caspase-3 and caspase-9 activation were measured to analyze the protective effects of tetrahydropalmatine against learning and memory impairment. As presented in Fig. 7, activation of caspase-3 and caspase-9 were higher in ketamine-induced mice compared with in the control group. Conversely, tetrahydropalmatine significantly inhibited caspase-3 and caspase- 9 activation in ketamine-induced mice (Fig. 7).

Protective effects of tetrahydropalmatine against GFAP, cytochrome $c$ and $P L C-\gamma 1$ protein expression in ketamine-induced mice. The present study aimed to determine the effects of tetrahydropalmatine on GFAP, cytochrome $c$ and PLC- $\gamma 1$ expression in ketamine-induced mice. As presented in Fig. 8, the protein expression levels of GFAP, cytochrome $c$ and PLC- $\gamma 1$ were significantly higher in ketamine-induced mice compared with in the control group. However, treatment with tetrahydropalmatine significantly suppressed GFAP, cytochrome $c$ and PLC- $\gamma 1$ protein expression in ketamine-induced mice (Fig. 8).

\section{Discussion}

A previous clinical retrospective cohort study indicated that general anesthesia administered to children $<4$ years old may be considered a risk factor for long-term learning disabilities following surgery (11). Therefore, examination of the effects of anesthetic agents on juvenile brains during the developmental period has great significance (12). Ketamine, which is a non-competitive antagonist of the NMDA receptor, is a commonly used intravenous anesthetic drug, and has been in use for nearly half a century and is widely used in pediatric surgery (13). However, its use is controversial due to the reported ketamine-induced reduction in juvenile learning and memory function (14). In addition, ketamine has been reported to induce schizophrenia-like behaviors and oxidative damage in mice (15). The present study demonstrated that tetrahydropalmatine effectively inhibited ketamine-induced increases in escape latency and mean path length, and reversed the ketamine-induced decreases in percentage of time spent in the target quadrant and number of times crossing the platform.

Oxidative stress has been reported to be an important cause of nerve cell damage (16). In addition, it has been demonstrated that the abnormal deposition of amyloid- $\beta(\mathrm{A} \beta)$ may induce oxidative stress, which leads to the loss of synaptic function and neuronal metabolism barrier, which serves a 

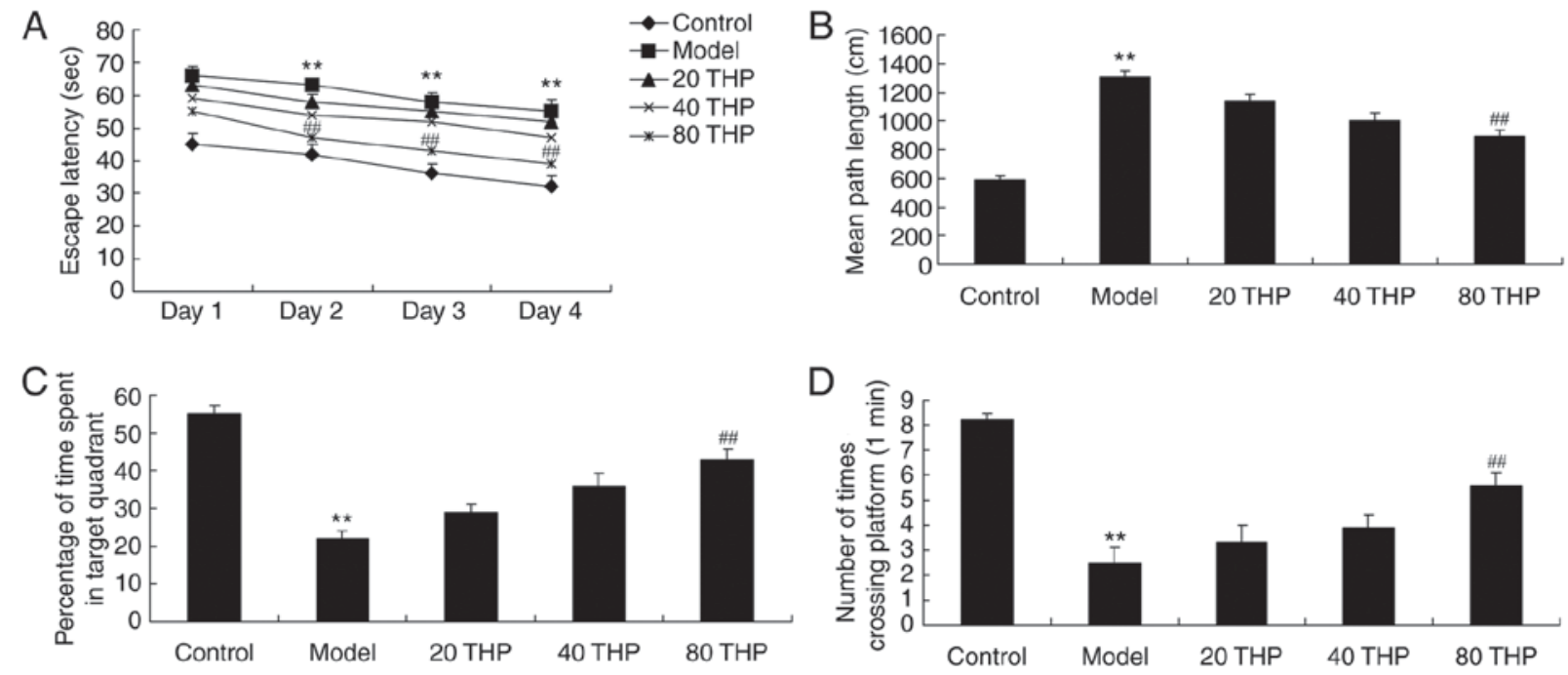

Figure 2. Protective effects of THP against ketamine-induced learning and memory impairment in mice. (A) Escape latency, (B) mean path length, (C) percentage of time spent in the target quadrant and (D) number of times crossing the platform. ${ }^{* *} \mathrm{P}<0.05$ vs. the control group; ${ }^{\# \#} \mathrm{P}<0.05$ vs. the model group. THP, tetrahydropalmatine.
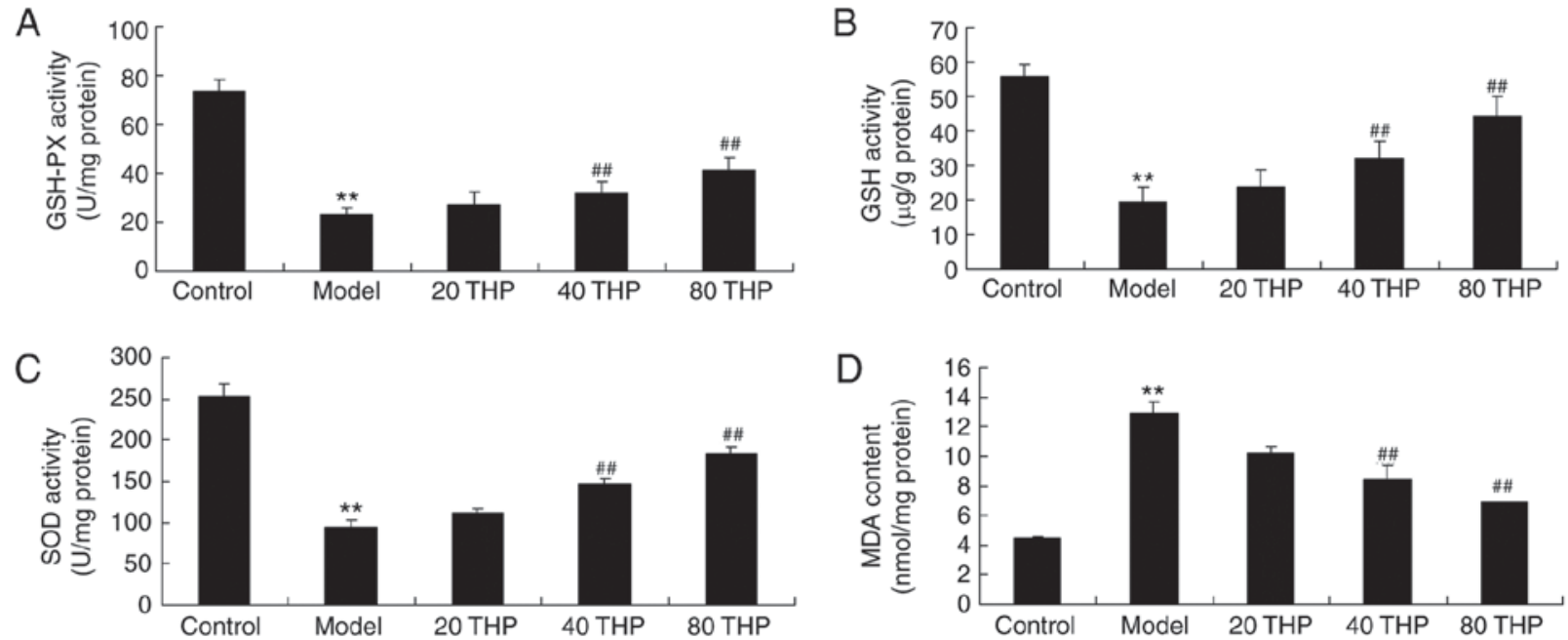

Figure 3. Protective effects of THP against oxidative stress in ketamine-induced mice. Effects of THP on (A) GSH-PX, (B) GSH, (C) SOD and (D) MDA activities in mice treated with ketamine. ${ }^{* *} \mathrm{P}<0.05$ vs. the control group; ${ }^{\# \#} \mathrm{P}<0.05$ vs. the model group. GSH, glutathione; GSH-PX, GSH-peroxidase; MDA, malondialdehyde; SOD, superoxide dismutase; THP, tetrahydropalmatine.

key role in the pathogenesis of learning and memory impairment (17). Elevated levels of oxidative stress in brain tissues induce injury to neurons (18). The present study demonstrated that tetrahydropalmatine significantly increased GSH-PX, GSH and SOD activity, inhibited MDA activity, and decreased TNF- $\alpha$, IL- $1 \beta$ and IL- 6 expression in ketamine-induced mice via the suppression of NF- $\kappa \mathrm{B}$ protein expression. Yu et al previously reported that tetrahydropalmatine can effectively attenuate irradiation-induced lung injury in the thoracic region through anti-apoptotic, antifibrotic and anti-inflammatory mechanisms (8).

In neurons, choline and acetyl coenzyme A are synthesized into ACh, which is catalyzed by choline acetyltransferase. $\mathrm{ACh}$ is stored in synaptic vesicles and, in response to stimulation, is released from cholinergic nerve endings (19). The postsynaptic membrane ACh receptor is known as the cholinergic receptor, of which there are two types: Muscarinic and nicotinic receptors, which are widely distributed in the central nervous system (20). The subsequent effects of ACh depend on the role of AChE in the synaptic cleft; AChE can hydrolyze ACh into choline and acetic acid, which has a very high catalytic activity, thus ensuring that the concentration of ACh declines rapidly (21). Therefore, AChE and choline acetyltransferase activities in the brain can indirectly reflect $\mathrm{ACh}$ content, and can thus be used to infer the functional status of the central cholinergic system (22). Furthermore, the present study indicated that tetrahydropalmatine significantly decreased the ketamine-induced increase in AChE activity and reversed the ketamine-induced decrease in ACh levels, demonstrating that tetrahydropalmatine protected nerve cell apoptosis in ketamine-induced mice. Qu et al clearly demonstrated that tetrahydropalmatine may protect against D-galactose-induced memory impairment in rats through AChE and ACh activity (23). 

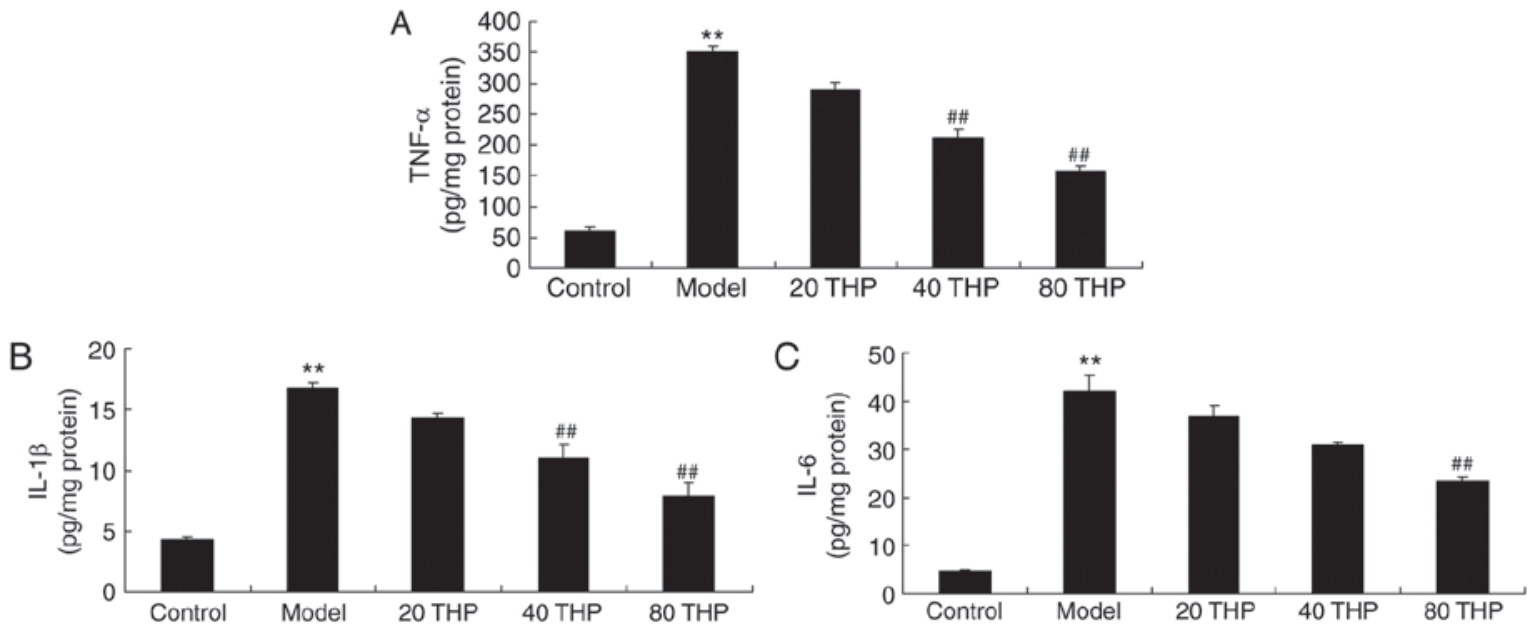

Figure 4. Protective effects of THP against inflammation in ketamine-induced mice. Effects of THP on (A) TNF- $\alpha$, (B) IL-1 $\beta$ and (C) IL-6 activities in mice treated with ketamine. ${ }^{* *} \mathrm{P}<0.05$ vs. the control group; ${ }^{\# / /} \mathrm{P}<0.05$ vs. the model group. IL, interleukin; THP, tetrahydropalmatine; TNF- $\alpha$, tumor necrosis factor- $\alpha$.
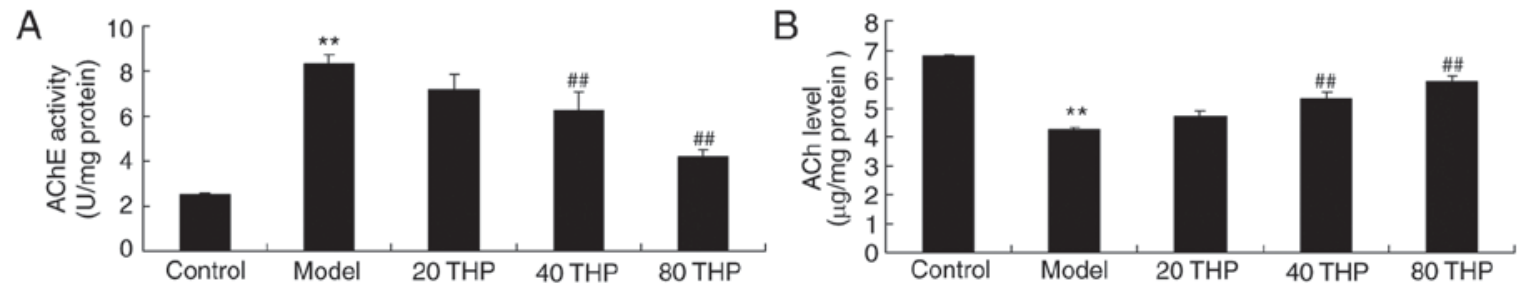

Figure 5. Protective effects of THP against ACh levels and AChE activity in ketamine-induced mice. Effects of THP on (A) AChE activity and (B) ACh levels in mice treated with ketamine. ${ }^{* *} \mathrm{P}<0.05$ vs. the control group; ${ }^{\# \#} \mathrm{P}<0.05$ vs. the model group. ACh, acetylcholine; AChE, acetylcholinesterase; THP, tetrahydropalmatine.
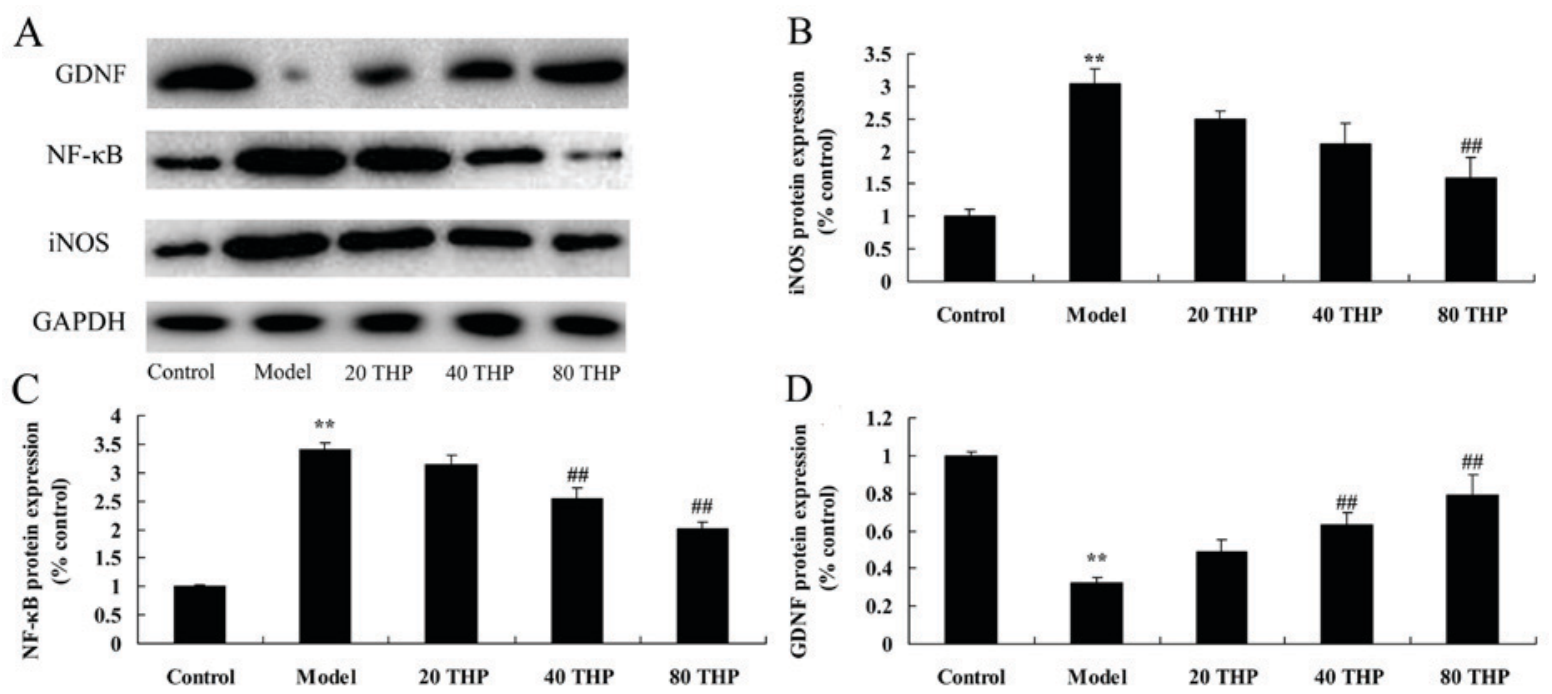

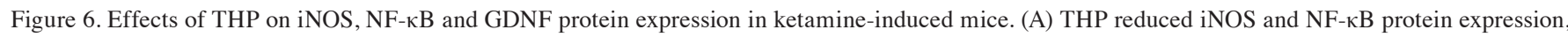
and induced GDNF protein expression in ketamine-induced mice, as determined using western blot analysis. (B-D) Protein expression levels of iNOS, NF- $\mathrm{kB}$ and GDNF in mice treated with ketamine were statistically analyzed. ${ }^{* *} \mathrm{P}<0.05$ vs. the control group; ${ }^{\# \#} \mathrm{P}<0.05$ vs. the model group. GDNF, glial cell-derived neurotrophic factor; iNOS, inducible nitric oxide synthase; NF- $\mathrm{BB}$, nuclear factor- $\mathrm{kB}$; THP, tetrahydropalmatine.

Learning and memory are brain functions that are indispensable to life (24). Learning is a neurological process that refers to the acquisition of novel information, whereas memory refers to the process by which obtained information is stored, organized and re-acquired by learning experiences in the brain. These two processes are interdependent; cognitive ability is a very important factor for learning and memory, and also an important factor associated with intelligence (20). The hippocampus is the main part of the brain associated with cognitive function. A previous 

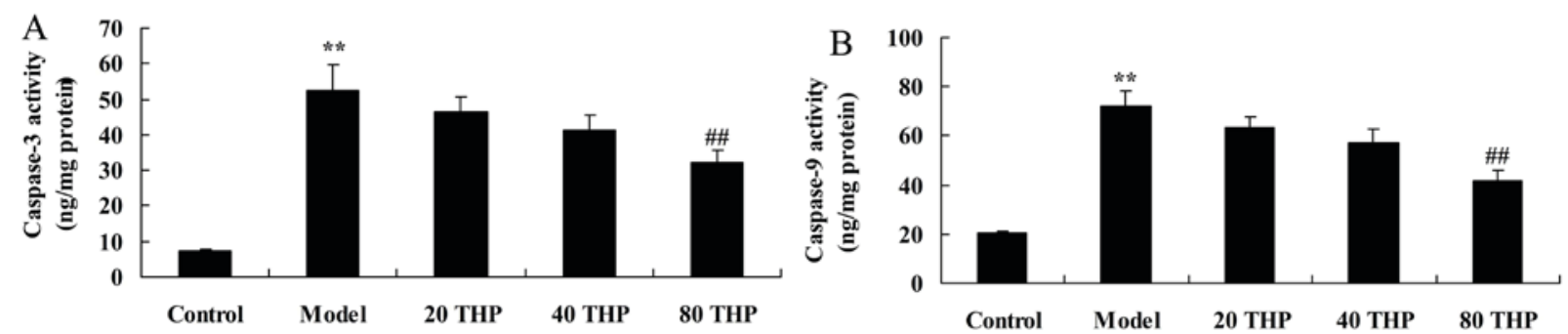

Figure 7. Protective effects of THP against caspase- 3 and caspase- 9 activation in ketamine-induced mice. THP protected against (A) caspase- 3 and (B) caspase- 9 activation in mice treated with ketamine. ${ }^{* *} \mathrm{P}<0.05$ vs. the control group; ${ }^{\# \#} \mathrm{P}<0.05$ vs. the model group. THP, tetrahydropalmatine.
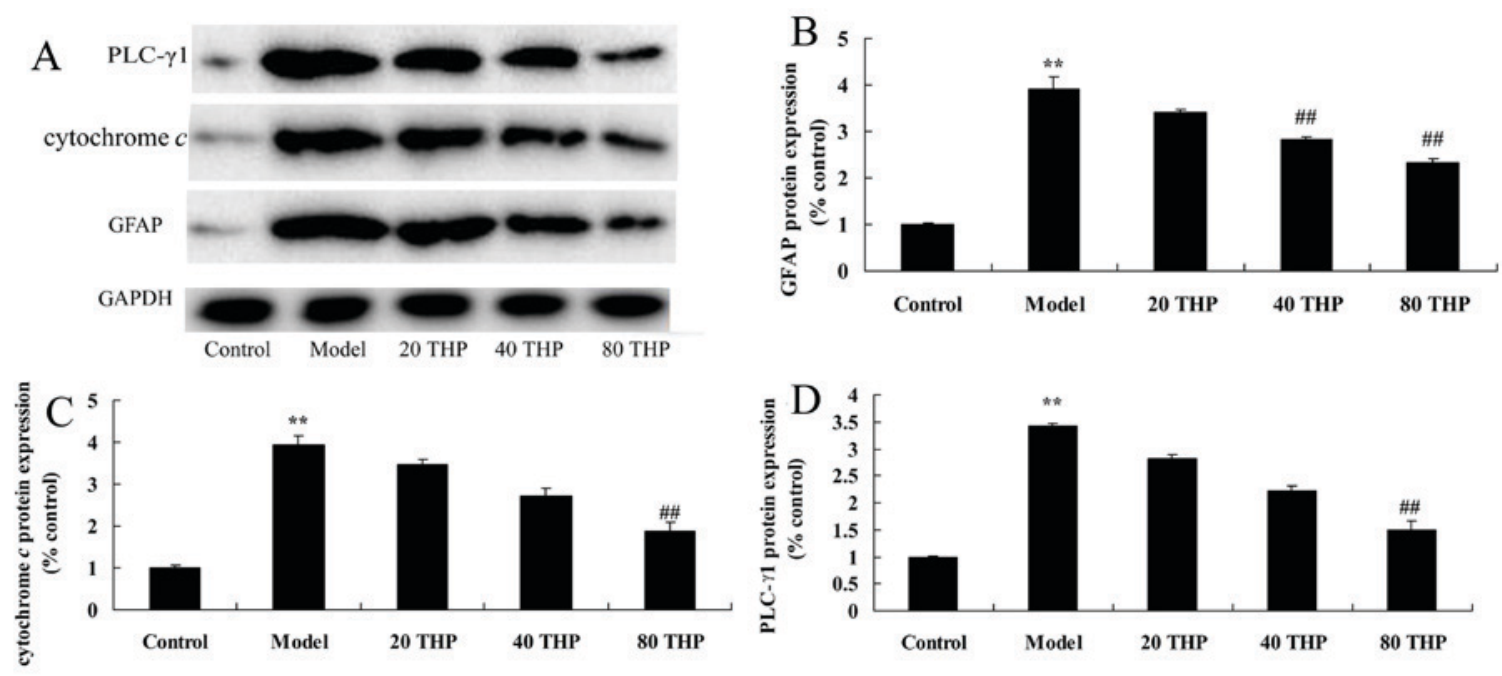

Figure 8. Protective effects of THP against GFAP, cytochrome $c$ and PLC- $\gamma 1$ protein expression in ketamine-induced mice. (A) THP protected against GFAP, cytochrome $c$ and PLC- $\gamma 1$ protein expression, as determined using western blot analysis. (B-D) Protein expression levels of GFAP, cytochrome $c$ and PLC- $\gamma 1$ in mice treated with ketamine were statistically analyzed. ${ }^{* *} \mathrm{P}<0.05$ vs. the control group; ${ }^{\# \# /} \mathrm{P}<0.05$ vs. the model group. GFAP, glial fibrillary acidic protein; PLC- $\gamma 1$, phospholipase C- $\gamma 1$; THP, tetrahydropalmatine.

study reported that the hippocampal CA1 region serves an important role in speech recognition, and declarative learning and memory (24). The present study revealed that tetrahydropalmatine significantly inhibited caspase-3 and caspase-9 activation in ketamine-induced mice. In addition, Yu et al demonstrated that tetrahydropalmatine may protect endothelial cells against gamma-irradiation injury via caspase-3 activation and cytochrome $c$ (7). The results of the present study demonstrated that tetrahydropalmatine may inhibit nerve cell apoptosis in ketamine-induced mice via the caspases signaling pathway.

B-cell lymphoma 2 (Bcl-2) and caspase-3 are two important members of the protein family that regulates cell apoptosis, in particular the role of these proteins in the regulation of brain cell apoptosis has been confirmed (25). Previous studies have reported that $\mathrm{Bcl}-2$ expression is closely associated with cell survival, and an increase in Bcl-2 expression in the brain may reduce infarct size and protect nerve cells $(25,26)$. In addition, it has been demonstrated, using an ischemia-reperfusion model, that lateral ventricle injection with caspase-3 inhibitors not only reduces caspase- 3 activity, but also significantly reduces infarct size and apoptosis (27). Caspase-3 is a downstream regulating protein of $\mathrm{Bcl}-2$, which is the originating factor for triggering apoptosis, and $\mathrm{Bcl}-2$ overexpression can effectively inhibit caspase-3 activation and apoptosis (27).
The specific enzyme of Bcl-2 requires caspase- 3 activation in the cell body for cell apoptosis; apoptosis inducing factor and cytochrome $c$ can activate DNA damage, leading to cascade activation of the caspase family, which induces apoptosis (28). The results of the present study suggested that tetrahydropalmatine may significantly suppress cytochrome $c$ protein expression in ketamine-induced mice. These data are consistent with the results of Yu et al (29), which indicated that tetrahydropalmatine protects rat pulmonary endothelial cells from irradiation-induced apoptosis by inhibiting cytochrome $c$ and PLC- $\gamma 1$.

GDNF is a newly-discovered neurotrophic factor, which was initially detected in rat glioblastoma. GDNF is a member of the transforming growth factor $\beta$ superfamily, which is mainly secreted by glial cells, and is expressed in the granule cells of the striatum, thalamic nuclei, hippocampus, cingulate gyrus and olfactory bulb, where it exerts a wide range of nutritional functions in various central nerve cells (30). At present, it is the only biological factor that can both resist neuronal apoptosis and prevent tissue atrophy of the nerve cell body (31). In addition, GDNF serves an important role in the cognitive functions of learning and memory; in a mouse model in which GDNF expression was knocked down, hippocampal synaptic transmission was abnormal and water maze performance was impaired (32). In this study, it was demonstrated that 
tetrahydropalmatine significantly increased GDNF protein expression in ketamine-induced mice.

GFAP is an important cytoskeletal protein for astrocyte synthesis, which is now recognized as a characteristic astrocyte marker (33). Diabetes can affect astrocytes, resulting in alterations in GFAP expression. A previous study revealed that in diabetic rats, the expression of GFAP is decreased in the rat cortex, hippocampus and cerebellum, resulting in a decrease in the generation of blood vessels, the blood-brain barrier and the change in LTP, eventually leading to learning and memory dysfunction (33). It has also been reported that with the long-term stimulation of hyperglycemia, learning and memory functions in rats are gradually decreased, accompanied by the increased expression of GFAP in hippocampus; these results indicated that astrocytes are associated with anesthesia-induced cognitive dysfunction $(34,35)$. The results of the present study indicated that tetrahydropalmatine significantly reduced GFAP protein expression in ketamine-induced mice. In addition, $\mathrm{Qu}$ et al clearly demonstrated that tetrahydropalmatine may protect against D-galactose-induced memory impairment through the inhibition of GFAP expression in rats (23).

PLC $\gamma 1$ is a member of the PLC serine/threonine family; the phosphorylation of tyrosine 783 results in its activation, signal transmission, and finally its corresponding cellular effect (36). In the nervous system epileptic seizures in mice may be significantly inhibited following induction of the tropomyosin receptor kinase B/PLC $\gamma 1$ signaling pathway; similarly, PLC $\gamma 1$ affects the structural plasticity of sensory neurons in the vestibular system and neuronal dendrite formation in the middle of the olfactory bulb (37). In cerebellar neurons and cortical neurons, PLC $\gamma 1$ activity is associated with the release of brain-derived neurotrophic factor-induced glutamate (38). In the present study, the results indicated that tetrahydropalmatine significantly suppressed PLC- $\gamma 1$ protein expression in ketamine-induced mice, thus suggesting that PLC- $\gamma 1$ may serve an important role in the effects of tetrahydropalmatine on ketamine-induced toxicity.

In conclusion, the present study clearly demonstrated that tetrahydropalmatine protects against ketamine-induced learning and memory impairment in mice. In addition, it was indicated that the protective effects of tetrahydropalmatine on learning and memory impairment were associated with antioxidative, anti-inflammatory and anti-apoptotic mechanisms. However, further studies are required to clarify the neurobiological mechanisms.

\section{References}

1. Li J, Yu Y, Wang B, Wu H, Xue G and Hou Y: Selective regulation of neurosteroid biosynthesis under ketamine-induced apoptosis of cortical neurons in vitro. Mol Med Rep 13: 1586-1592, 2016.

2. Liu JR, Baek C, Han XH, Shoureshi P and Soriano SG: Role of glycogen synthase kinase-3 $\beta$ in ketamine-induced developmental neuroapoptosis in rats. Br J Anaesth 110 (Suppl 1): Si3-Si9, 2013.

3. Cetin N, Suleyman B, Altuner D, Kuyrukluyildiz U, Ozcicek F, Coskun R, Kurt N and Suleyman H: Effect of disulfiram on ketamine-induced cardiotoxicity in rats. Int J Clin Exp Med 8: 13540-13547, 2015.

4. Liu JR, Liu Q, Li J, Baek C, Han XH, Athiraman U and Soriano SG: Noxious stimulation attenuates ketamine-induced neuroapoptosis in the developing rat brain. Anesthesiology 117: 64-71, 2012.
5. D'Souza DC, Ahn K, Bhakta S, Elander J, Singh N, Nadim H, Jatlow P, Suckow RF, Pittman B and Ranganathan M: Nicotine fails to attenuate ketamine-induced cognitive deficits and negative and positive symptoms in humans: Implications for schizophrenia. Biol Psychiatry 72: 785-794, 2012.

6. Huang S, Dai Y, Zhang Z, Hao W and Chen H: Docosahexaenoic acid intake ameliorates ketamine-induced impairment of spatial cognition and learning ability in ICR mice. Neurosci Lett 580: 125-129, 2014.

7. Yu J, Piao BK, Pei YX, Qi X and Hua BJ: Protective effects of tetrahydropalmatine against gamma-radiation induced damage to human endothelial cells. Life Sci 87: 55-63, 2010.

8. Yu J, Che J, Liu L, Yang F, Zhu X and Cao B: Tetrahydropalmatine attenuates irradiation induced lung injuries in rats. Life Sci 153: 74-81, 2016

9. Zhao Y, Liang A, Zhang Y, Li C, Yi Y and Nilsen OG: Impact of Tetrahydropalmatine on the pharmacokinetics of probe drugs for CYP1A2, 2D6 and 3A isoenzymes in beagle dogs. Phytother Res 30: 906-914, 2016.

10. Koffler SP, Hampstead BM, Irani F, Tinker J, Kiefer RT, Rohr P and Schwartzman RJ: The neurocognitive effects of 5 day anesthetic ketamine for the treatment of refractory complex regional pain syndrome. Arch Clin Neuropsychol 22: 719-729, 2007.

11. Dahan A, Olofsen E, Sigtermans M, Noppers I, Niesters M, Aarts L, Bauer M and Sarton E: Population pharmacokinetic-pharmacodynamic modeling of ketamine-induced pain relief of chronic pain. Eur J Pain 15: 258-267, 2011.

12. Hashimoto K: A BDNF Val66Met Polymorphism and ketamine-induced rapid antidepressant action. Clin Psychopharmacol Neurosci 10: 59-60, 2012.

13. Zhou W, Wang N, Yang C, Li XM, Zhou ZQ and Yang JJ: Ketamine-induced antidepressant effects are associated with AMPA receptors-mediated upregulation of mTOR and BDNF in rat hippocampus and prefrontal cortex. Eur Psychiatry 29: 419-423, 2014.

14. Li J, Wang B, Wu H, Yu Y, Xue G and Hou Y: $17 \beta$-estradiol attenuates ketamine-induced neuroapoptosis and persistent cognitive deficits in the developing brain. Brain Res 1593: 30-39, 2014.

15. Ben-Azu B, Aderibigbe AO, Ajayi AM and Iwalewa EO: Neuroprotective effects of the ethanol stem bark extracts of Terminalia ivorensis in ketamine-induced schizophrenia-like behaviors and oxidative damage in mice. Pharm Biol 54: 2871-2879, 2016

16. Li J, Liu CN, Wei N, Li XD, Liu YY, Yang R and Jia YJ: Protective effects of BAY 73-6691, a selective inhibitor of phosphodiesterase 9, on amyloid-beta peptides-induced oxidative stress in in-vivo and in-vitro models of Alzheimer's disease. Brain Res 1642: 327-335, 2016.

17. Liu KM, Chuang SM, Long CY, Lee YL, Wang CC, Lu MC, Lin RJ, Lu JH, Jang MY, Wu WJ, et al: Ketamine-induced ulcerative cystitis and bladder apoptosis involve oxidative stress mediated by mitochondria and the endoplasmic reticulum. Am J Physiol Renal Physiol 309: F318-F331, 2015.

18. de Araujo FY, de Oliveira GV, Gomes PX, Soares MA, Silva MI, Carvalho AF, de Moraes MO, de Moraes ME, Vasconcelos SM, Viana GS, et al: Inhibition of ketamine-induced hyperlocomotion in mice by the essential oil of Alpinia zerumbet: Possible involvement of an antioxidant effect. J Pharm Pharmacol 63: 1103-1110, 2011

19. Wang Q, Sun LH, Jia W, Liu XM, Dang HX, Mai WL, Wang N, Steinmetz A, Wang YQ and Xu CJ: Comparison of ginsenosides $\mathrm{Rg} 1$ and $\mathrm{Rb} 1$ for their effects on improving scopolamine-induced learning and memory impairment in mice. Phytother Res 24: $1748-1754,2010$

20. Hall JM and Savage LM: Exercise leads to the re-emergence of the cholinergic/nestin neuronal phenotype within the medial septum/diagonal band and subsequent rescue of both hippocampal ACh efflux and spatial behavior. Exp Neurol 278: 62-75, 2016.

21. Chen HW, He XH, Yuan R, Wei BJ, Chen Z, Dong JX and Wang J: Sesquiterpenes and a monoterpenoid with acetylcholinesterase (AchE) inhibitory activity from Valeriana officinalis var. Latiofolia in vitro and in vivo. Fitoterapia 110: 142-149, 2016.

22. Xu NG, Xiao ZJ, Zou T and Huang ZL: Ameliorative effects of physcion 8-O- $\beta$-glucopyranoside isolated from Polygonum cuspidatum on learning and memory in dementia rats induced by Abeta1-40. Pharm Biol 53: 1632-1638, 2015. 
23. Qu Z, Zhang J, Yang H, Huo L, Gao J, Chen H and Gao W: Protective effect of tetrahydropalmatine against d-galactose induced memory impairment in rat. Physiol Behav 154: 114-125, 2016.

24. Wan D, Xue L, Zhu H and Luo Y: Catalpol induces neuroprotection and prevents memory dysfunction through the cholinergic system and BDNF. Evid Based Complement Alternat Med 2013: $134852,2013$.

25. Li XY, Xu L, Liu CL, Huang LS and Zhu XY: Electroacupuncture intervention inhibits the decline of learning-memory ability and overex- pression of cleaved caspase- 3 and bax in hippocampus induced by isoflurane in APPswe/PS 1. Zhen Ci Yan Jiu 41: 24-30, 2016.

26. Li M, Peng J, Wang MD, Song YL, Mei YW and Fang Y: Passive movement improves the learning and memory function of rats with cerebral infarction by inhibiting neuron cell apoptosis. Mol Neurobiol 49: 216-221, 2014.

27. Xian YF, Mao QQ, Wu JC, Su ZR, Chen JN, Lai XP, Ip SP and Lin ZX: Isorhynchophylline treatment improves the amyloid- $\beta$-induced cognitive impairment in rats via inhibition of neuronal apoptosis and tau protein hyperphosphorylation. J Alzheimers Dis 39: 331-346, 2014.

28. Doniselli N, Monzeglio E, Dal Palù A, Merli A and Percudani R: The identification of an integral membrane, cytochrome $\mathrm{c}$ urate oxidase completes the catalytic repertoire of a therapeutic enzyme. Sci Rep 5: 13798, 2015.

29. Yu J,Zhao L, Liu L, Yang F,Zhu X and Cao B: Tetrahydropalmatine protects rat pulmonary endothelial cells from irradiation-induced apoptosis by inhibiting oxidative stress and the calcium sensing receptor/phospholipase C- $\gamma 1$ pathway. Free Radic Res 50 611-626, 2016.

30. Miyazaki H, Okuma Y, Nomura J, Nagashima K and Nomura Y: Age-related alterations in the expression of glial cell line-derived neurotrophic factor in the senescence-accelerated mouse brain J Pharmacol Sci 92: 28-34, 2003.
31. Zhang J, Tan H, Jiang W and Zuo Z: Amantadine alleviates postoperative cognitive dysfunction possibly by increasing glial cell line-derived neurotrophic factor in rats. Anesthesiology 121: 773-785, 2014.

32. Pertusa M, Garcia-Matas S, Mammeri H, Adell A, Rodrigo T, Mallet J, Cristòfol R, Sarkis C and Sanfeliu C: Expression of GDNF transgene in astrocytes improves cognitive deficits in aged rats. Neurobiol Aging 29: 1366-1379, 2008.

33. Sudo G, Kagawa T, Kokubu Y, Inazawa $J$ and Taga $T$ : Increase in GFAP-positive astrocytes in histone demethylase GASC1/KDM4C/JMJD2C hypomorphic mutant mice. Genes Cells 21: 218-225, 2016.

34. Silva AF, Aguiar MS, Carvalho OS, Santana Lde N, Franco EC, Lima RR, Siqueira NV, Feio RA, Faro LR and Gomes-Leal W: Hippocampal neuronal loss, decreased GFAP immunoreactivity and cognitive impairment following experimental intoxication of rats with aluminum citrate. Brain Res 1491: 23-33, 2013.

35. Chuang CM, Hsieh CL, Lin HY and Lin JG: Panax Notoginseng Burk attenuates impairment of learning and memory functions and increases ED1, BDNF and beta-secretase immunoreactive cells in chronic stage ischemia-reperfusion injured rats. Am J Chin Med 36: 685-693, 2008.

36. Gu B, Huang YZ, He XP, Joshi RB, Jang W and McNamara JO: A peptide uncoupling BDNF receptor TrkB from phospholipase $\mathrm{C} \gamma 1$ prevents epilepsy induced by status epilepticus. Neuron 88 : 484-491, 2015.

37. Zhou L, Martinez SJ, Haber M, Jones EV, Bouvier D, Doucet G, Corera AT, Fon EA, Zisch AH and Murai KK: EphA4 signaling regulates phospholipase Cgammal activation, cofilin membrane association and dendritic spine morphology. J Neurosci 27: 5127-5138, 2007.

38. Cortese GP, Barrientos RM, Maier SF and Patterson SL: Aging and a peripheral immune challenge interact to reduce mature brain-derived neurotrophic factor and activation of TrkB, PLCgamma1 and ERK in hippocampal synaptoneurosomes. J Neurosci 31: 4274-4279, 2011. 